\title{
Reparación laparoscópica de hernia de Spiegel. ¿Cómo lo hacemos nosotros?
}

\author{
Laparoscopic Spiegel hernia \\ repair. How we do it? \\ Jorge Farell Rivas, ${ }^{\star}$ Noé Pérez Carrillo, ${ }^{*}$ Ana Paula Ruiz Funes Molina, ${ }^{\ddagger}$ \\ Nubia Andrea Ramírez Buensuceso Conde* \\ * Cirugía General. \\ ‡ Cirugía Endoscópica.
}

Hospital Central Sur de Alta Especialidad de Petróleos Mexicanos, Ciudad de México.

RESUMEN

El abordaje laparoscópico transabdominal preperitoneal es un tratamiento quirúrgico eficaz para la hernia de Spiegel, esta técnica de mínimo acceso permite identificar la ubicación exacta del defecto anatómico y evita la necesidad de realizar incisiones cutáneas extensas. Permite la colocación de una malla retromuscular con menor posibilidad de recurrencia así como una recuperación más rápida. Presentamos los principios quirúrgicos técnicos que hemos considerado esenciales para una hernioplastia exitosa.

Palabras clave: Laparoscopía, hernia Spiegel, técnica.
ABSTRACT

Laparoscopic transabdominal preperitoneal approach is an effective surgical treatment for Spiegelian hernia. Minimally invasive technique allows to identify the exact location of the anatomical defect and avoids the need for extensive skin incisions. It allows a less traumatic and extensive dissection offered by conventional approach, and adds the possibility to apply a retromuscular mesh with big overlap. We present the technical surgical principles we have found essential for a successful repair.

Keywords: Laparoscopic, hernia Spiegel, technique.

\section{INTRODUCCIÓN}

La hernia de Spiegel, también Ilamada «hernia ventral lateral espontánea» o «hernia de la línea semilunar», ${ }^{1}$ es causada por una debilidad de la zona de Spiegel. Esta área se encuentra entre el recto abdominal y la línea semilunar, formada por el músculo transverso y la aponeurosis del oblicuo interno. ${ }^{2}$ Sin embargo, hay au- tores que también hacen referencia a causas adquiridas como hernias posincisionales. ${ }^{3}$ Su frecuencia es baja, de 0.1 a $2.5 \%$ del total de hernias de pared abdominal, y se presentan con mayor frecuencia en mujeres. Más de $90 \%$ se encuentran en el Ilamado «cinturón spiegeliano de hernias», una zona transversa de $6 \mathrm{~cm}$ de ancho localizada arriba del plano interespinoso. Ésta es la zona donde la fascia de Spiegel pasa de estar

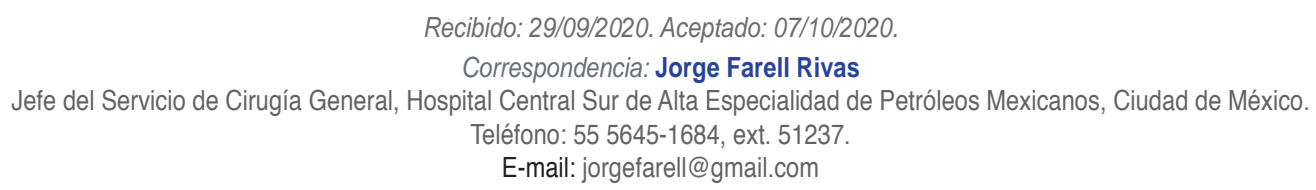

Citar como: Farell RJ, Pérez CN, Ruiz FMAP, Ramírez BCNA. Reparación laparoscópica de hernia de Spiegel. ¿Cómo lo hacemos nosotros? Rev Mex Cir Endoscop. 2020; 21 (4): 200-205. https://dx.doi.org/10.35366/101219 
compuesta por una sola capa resistente y se divide en dos hojas separadas, lo que crea una debilidad en la pared. Se han considerado varios factores que pudieran contribuir a su etiología como aumento de la presión intraabdominal, deterioro de la pared abdominal, alteraciones de colágeno, edad, obesidad, embarazos múltiples, pérdida de peso, enfermedad pulmonar obstructiva crónica, tabaquismo, tos crónica, traumatismo cerrado y cirugías abdominales previas, con especial riesgo en la cirugía laparoscópica. Los casos congénitos se explican por un desarrollo embriológico anormal de la pared muscular. ${ }^{4}$ Su diagnóstico requiere de alto nivel de sospecha, dada la falta de síntomas y signos consistentes. El síntoma predominante es el dolor seguido de un tumor palpable. ${ }^{5}$ La confirmación del diagnóstico se realiza mediante un ultrasonido de la pared abdominal o una tomografía computarizada (TC) de abdomen, de preferencia en Valsalva.

Para el manejo de la hernia de Spiegel se utilizan abordajes tanto abiertos como laparoscópicos. En cirugía endoscópica se ha reportado mayor experiencia mediante un abordaje transabdominal. ${ }^{1}$ El cierre laparoscópico es seguro, rentable y la técnica es altamente reproducible. ${ }^{6}$ Preferimos el abordaje transabdominal preperitoneal (TAPP) por todas las ventajas descritas anteriormente, además permite evaluar la viabilidad del contenido del saco, aunado a la posibilidad de realizar una cobertura con malla.

\section{PREPARACIÓN DEL PACIENTE}

Para tener los mejores resultados posibles en la realización de la cirugía, siempre es útil un periodo de prehabilitación de cuatro semanas, además preferimos evaluar algunos aspectos antes del procedimiento de acuerdo con las guías STRONG del American College of Surgeons, ${ }^{7}$ atendiendo cuatro áreas: Nutrición, tabaquismo, glucemia y manejo de medicamentos. Para la cirugía electiva es importante asegurarse de que los pacientes dejen de fumar y que su índice de masa corporal sea el menor posible. Para confirmar el diagnóstico, se realiza una exploración física de la pared abdominal y en todos los casos se solicita una TC abdominal (Figura 1).

\section{PREOPERATORIO}

1. Se requiere un ayuno de ocho horas, según protocolo institucional.

2. El riesgo de tromboembolismo se evalúa con la escala de Caprini para implementar profilaxis mecánica o farmacológica de acuerdo al riesgo. ${ }^{8}$

3. Para la profilaxis con antibióticos se utilizan las pautas de las guías antimicrobianas de Stanford 2019, donde el agente preferido es una cefalosporina. Utilizamos cefalosporinas de segunda o tercera generación, y si existe alergia a betalactámicos, utilizamos clindamicina. $^{9}$

4. En casos seleccionados, como defectos herniarios grandes o hernias recurrentes, se deja una sonda urinaria durante toda la cirugía para mantener la vejiga vacía.

5. Se aplica un antiséptico comercial a base de clorhexidina al $2 \%$ en una presentación de 26, 10.5 o $1.5 \mathrm{~mL}$ en $70 \%$ de alcohol isopropílico en el campo quirúrgico de acuerdo con las pautas locales.
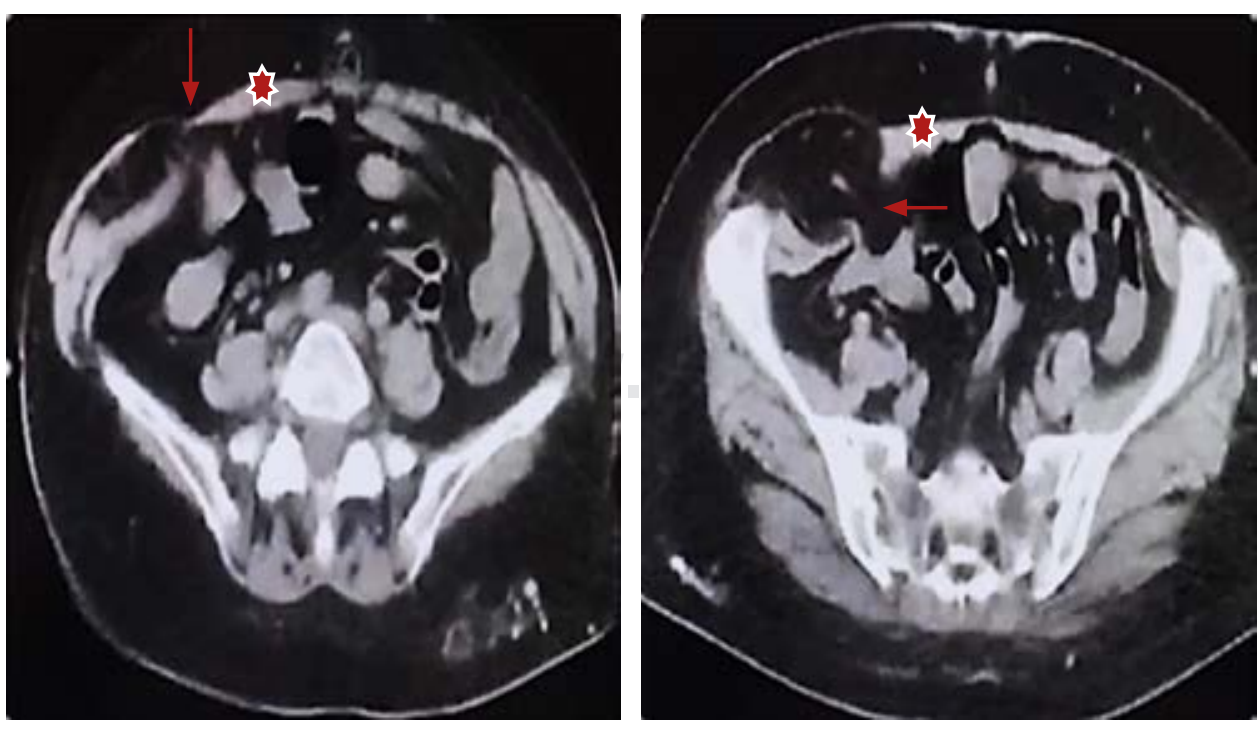

Figura 1:

Corte axial de tomografía computarizada que muestra el defecto en la línea semilunar derecha (flecha) correspondiente a una hernia de Spiegel, músculo recto anterior (asterisco). 

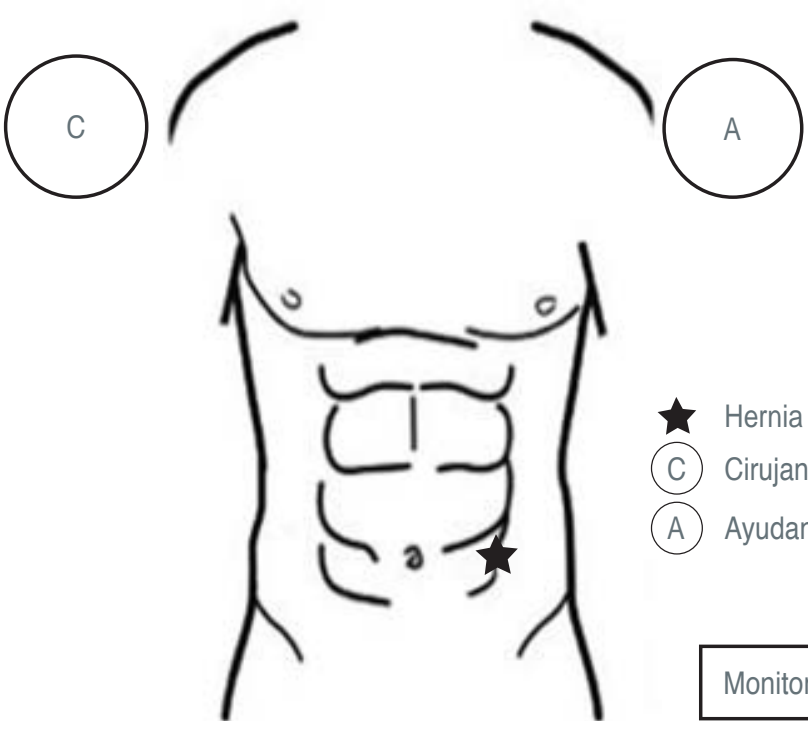

Hernia

(C) Cirujano

(A) Ayudante

Monitor

se insertan dos trocares de $5 \mathrm{~mm}$ accesorios, bajo visión directa (Figura 3).

\section{Creación del colgajo peritoneal y disección del saco herniario}

Previo a la disección del colgajo peritoneal, se intenta reducir el contenido de la hernia con tracción fina. Se crea un colgajo peritoneal similar al abordaje tipo TAPP utilizado para la reparación de hernia inguinal. ${ }^{11}$ Se inicia la creación de flap, al menos $4 \mathrm{~cm}$ proximal al defecto en sentido horizontal, esto sucede en el espacio preperitoneal, con disección roma o cortante, ya que es un espacio mayormente avascular. El saco herniario debe visualizarse con claridad y disecarse de manera suave (Figura 4), teniendo cuidado para evitar lesiones en los vasos epigástricos inferiores, la línea semilunar y el contenido del saco, sugerimos realizar la disección preperitoneal rodeando el saco y al final atacar el mismo y reducir el saco, este paso previo permite con mayor facilidad la resolución del caso (Figura 5).

\section{EQUIPO}

Dentro del equipo necesario se incluye:

1. Laparoscopio de $30^{\circ}$.

2. Pinzas atraumáticas e instrumentos de disección.

3. Dispositivo de energía bipolar o bisturí ultrasónico.

4. Tijera y portaagujas laparoscópico.

5. Sutura barbada o sutura no absorbible (polipropileno 0).

6. Malla de polipropileno macroporosa medianamente pesada (tamaño acorde a dimensiones del defecto herniario). ${ }^{10}$

\section{PROCEDIMIENTO}

\section{Acceso abdominal y colocación de puertos}

Después de la inducción de la anestesia general, se coloca al paciente en decúbito supino con ambos brazos pegados al cuerpo y se fija a la mesa quirúrgica. El equipo quirúrgico se sitúa de la siguiente manera: el cirujano se coloca del lado contralateral a la hernia, el primer ayudante se coloca a la altura de la cabeza del paciente (Figura 2).

El neumoperitoneo se obtiene mediante la técnica abierta de Hasson o con una aguja de Veress. Mediante un abordaje transabdominal preperitoneal (TAPP) se utilizan tres trocares (lente de $12 \mathrm{~mm}$ y dos accesorios de $5 \mathrm{~mm}$ ), se inserta un trocar de $12 \mathrm{~mm}$ en la región epigástrica o supraumbilical, al menos a $15 \mathrm{~cm}$ del defecto herniario. Posteriormente se realiza una laparoscopía diagnóstica y

\section{Cierre del defecto}

Una vez creado un espacio preperitoneal suficiente para la colocación de la malla se continúa con el cierre del defecto herniario, esta maniobra disminuye la recurrencia así como la incidencia en formación de seroma. ${ }^{12}$ El cierre del

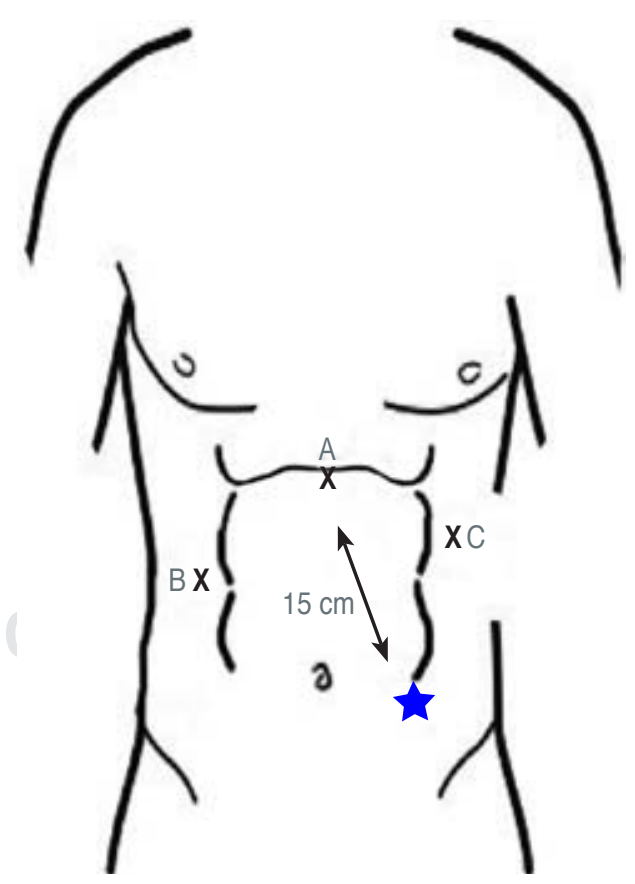

Figura 3: Colocación de trocares. (A) cámara, (B y C) trocares accesorios de $5 \mathrm{~mm}$, defecto herniario (estrella). 
Figura 4:

A) Vista laparoscópica del saco herniario y su contenido de epiplón.

B) Se realiza una disección suave del saco herniario.
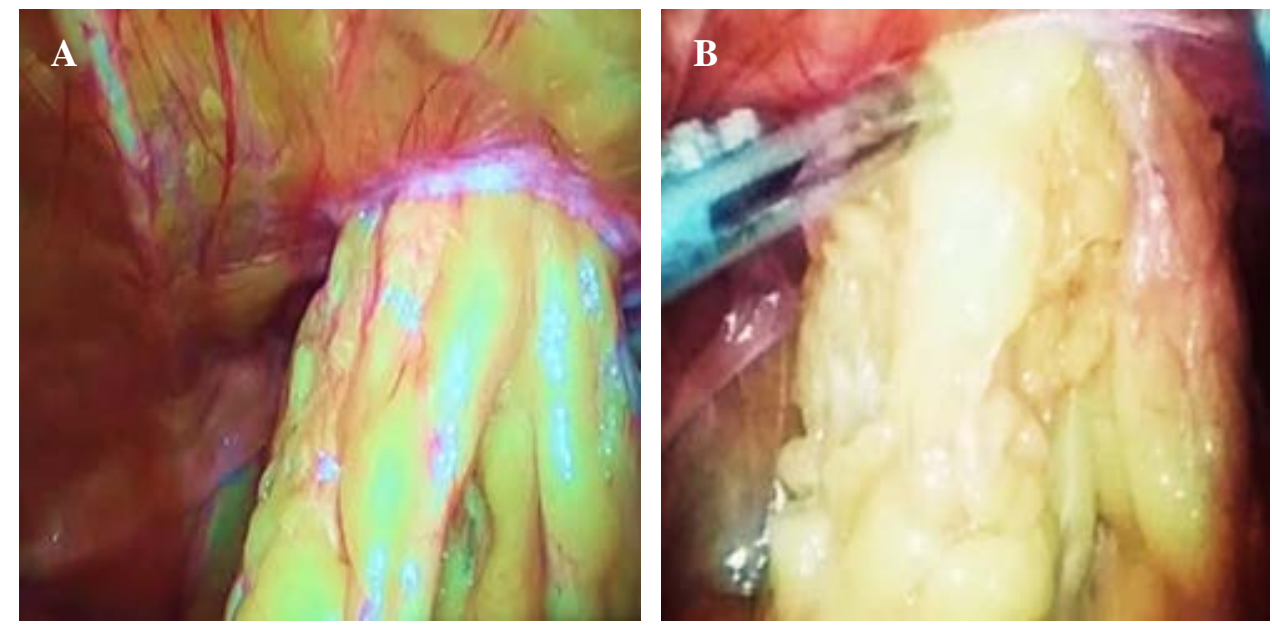

defecto herniario se puede realizar de múltiples formas; si se dispone de una sutura barbada de absorción lenta se obtiene más velocidad al cierre, un surgete simple o puntos simples en $\mathrm{X}$ son opciones fáciles de alcanzar.

\section{Fijación de la malla y cierre del peritoneo}

Es necesario crear suficiente espacio para la colocación de una malla que sobrepase en más de $4 \mathrm{~cm}$ cada uno de los bordes del defecto herniario ( ej. defecto de $4 \mathrm{~cm}$ requiere al menos una malla de $15 \times 15 \mathrm{~cm}$ ). Es nuestra preferencia la utilización de mallas de polipropileno macroporosas medianamente pesadas y su fijación con sutura absorbible intracorpórea en cuatro puntos cardinales (Figura 6). Otra alternativa para fijar la malla es con grapas absorbibles. El cierre peritoneal se realiza con una sutura continua simple con multifilamento absorbible 2-0 (Figura 7).

\section{Para finalizar}

El campo se examina para verificar hemostasia y la integridad del flap peritoneal, en caso de tener perforaciones en el mismo se sugiere reparar con puntos simples intracorpóreo para evitar la exposición de la malla al contenido abdominal. Los puertos se retiran bajo visión directa. Se cierra la fascia en los puertos que resultan en un defecto fascial mayor de $5 \mathrm{~mm}$ y por último, se cierra la piel con una sutura absorbible mediante puntos separados subdérmicos.

\section{MANEJO POSOPERATORIO}

Después de la cirugía, de acuerdo con el Programa de Recuperación Mejorada «SMART», por su siglas en inglés, de la Society of American Gastrointestinal and Endoscopic Surgeons (SAGES), ${ }^{13}$ evaluamos de forma rutinaria la náusea

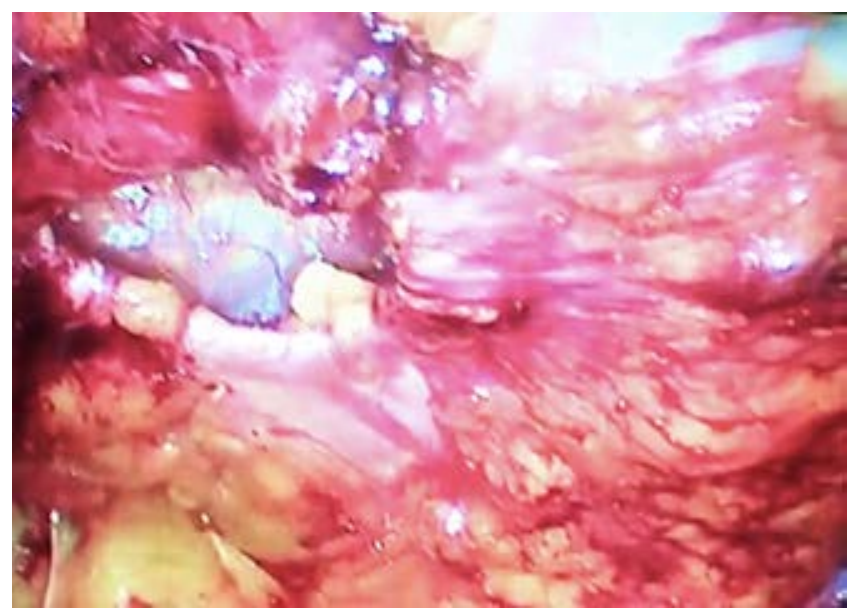

Figura 5: Defecto herniario.

y la emesis con la puntuación de la escala Apfel. Al final de la cirugía iniciamos un esquema de ondansetrón $8 \mathrm{mg}$ intravenoso cada ocho horas por un total de tres dosis. Otra consideración adicional sería el uso de fenotiazinas como la metoclopramida. Para manejo del dolor utilizamos $1 \mathrm{~g}$ de paracetamol cada seis horas, sumado a ketorolaco 30 mg cada ocho horas. Realizamos tromboprofilaxis farmacológica y/o mecánica según la puntuación del Caprini score en el posoperatorio.

Para prevenir el íleo, es necesaria una movilización temprana fuera de cama así como el inicio de dieta líquida y su progresión según la tolerancia en el primer día. Los pacientes se van a casa durante las primeras 24 horas después de finalizar la cirugía.

A la semana uno, al primer mes y a los seis meses, todos los pacientes acuden a su revisión clínica de seguimiento. Preguntamos a los pacientes si han experimentado algún 
problema posoperatorio (como infección de la herida, parestesia o dolor crónico). Se examinan en busca de evidencia clínica de seroma, hematoma o recurrencia.

\section{DISCUSION}

La hernia de Spiegel es una hernia poco frecuente de la pared abdominal, ${ }^{5}$ la particularidad anatómica resulta en un reto diagnóstico, lo cual es importante también al momento de la reparación laparoscópica con abordaje TAPP. Se recomienda el cierre primario de la fascia cuando los defectos herniarios son mayores de $1 \mathrm{~cm}$ en TAPP, ${ }^{14}$ esto ha demostrado disminución en la formación de seromas, disminución de infección del sitio quirúrgico y de la recurrencia de la hernia. ${ }^{11}$ Cuando se cierra la fascia es de utilidad tomar parte del saco herniario, ya que nos ayuda a disminuir el espacio muerto. Por otro lado, no recomendamos la cauterización de saco herniario, ya que al momento la evidencia científica es débil con pocos estudios que demuestren algún beneficio. ${ }^{11}$

La malla ideal depende en gran medida de su disponibilidad institucional, costo y preferencia del cirujano. Se recomienda el uso de mallas macroporosas de polipropileno medianamente pesadas por su bajo costo-complicaciones. La fijación de esta malla debe realizarse con sutura o fijadores absorbibles. En una revisión sistemática y metaanálisis Baker y colaboradores informan que la fijación con sutura de la malla se asoció con menor tasa de recurrencia de la hernia en comparación con tackers permanentes. ${ }^{15}$

\section{CONCLUSIONES}

El abordaje transabdominal preperitoneal laparoscópico es seguro para la reparación de hernias laterales ventrales como la hernia de Spiegel. En nuestra experiencia con este tipo de hernias las técnicas de mínimo acceso han mostrado un amplio beneficio en el manejo porque permiten una ubicación exacta del defecto anatómico, evitan la necesidad de incisiones cutáneas extensas y permiten

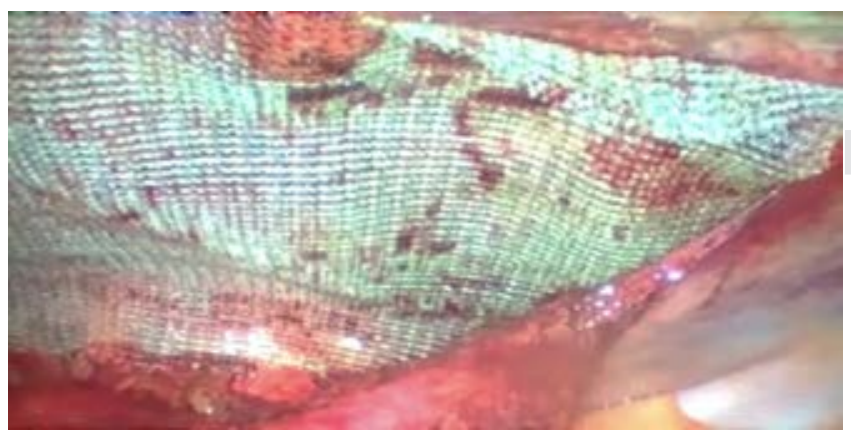

Figura 6: Malla fijada con puntos separados de polipropileno.

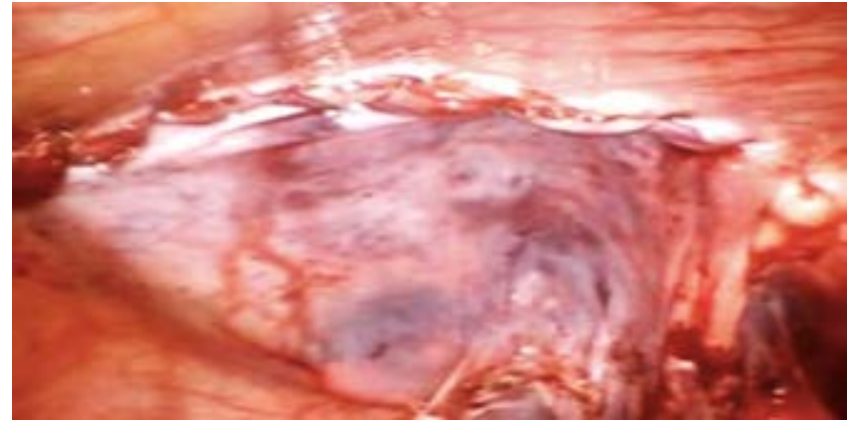

Figura 7: Cierre del peritoneo con surgete continuo.

disección menos traumática de los planos abdominales. La colocación de una malla de bajo costo en posición retromuscular produce menor recurrencia, menos dolor posoperatorio y una recuperación más rápida.

\section{REFERENCIAS}

1. Mittal T, Kumar V, Khullar R, Sharma A, Soni V, Baijal M et al. Diagnosis and management of Spigelian hernia: A review of literature and our experience. J Minim Access Surg. 2008; 4: 95-98.

2. Rankin A, Kostusiak M, Sokker A. Spigelian hernia: case series and review of the literature. Visc Med. 2019; 35: 133-136.

3. Polistina FA, Garbo, G, Trevisan P, Frego M. Twelve years of experience treating Spigelian hernia. Surgery [Internet]. 2015 [Access August 01, 2020]; 157: 547-550. Available in: https://doi.org/10.1016/j.surg.2014.09.027

4. Murillo ZA, Hernández LA, Puente EJ. Hernia de Spiegel, presentación de un caso y revisión de la literatura. Rev Mex Cir Endoscop. 2011; 12: 130-134.

5. Palanivelu C, Vijaykumar M, Jani KV, Rajan PS, Maheshkumaar GS, Rajapandian S. Laparoscopic transabdominal preperitoneal repair of spigelian hernia. JSLS. 2006; 10: 193-198.

6. Skouras C, Purkayastha S, Jiao L, Tekkis P, Darzi A, Zacharakis E. Laparoscopic management of spigelian hernias. Surg Laparosc Endosc Percutan Tech. 2011; 21: 76-81.

7. Mueller C, Compher C, Ellen DM; American Society for Parenteral and Enteral Nutrition (A.S.P.E.N.) Board of Directors. A.S.P.E.N. clinical guidelines: nutrition screening, assessment, and intervention in adults. JPEN J Parenter Enteral Nutr [Internet]. 2011 [Access August 01, 2020]; 35: 16-24. Available in: http://www.ncbi.nlm.nih.gov/ pubmed/21224430

8. Holubar M, Mui E, Deresinski S. Surgical antimicrobial prophylaxis guidelines. Stanford Antimicrobial Safety and Sustainability Program [Internet]. 2019 [Access August 06, 2020]. Available in: http://med.stanford. edu/bugsanddrugs/guidebook/_jcr_content/main/panel_ builder_584648957/panel_0/download_51076866/file.res/ SHC_SürgProphylaxisGuidēlines.pdf 
9. SAGES/SMART Enhanced Recovery Program [Internet]. Society of American Gastrointestinal and Endoscopic Surgeons. 2020 [Access August 02, 2020]. Available in: https://www.sages.org/smart-enhanced-recovery-program/

10. Baylón K, Rodríguez-Camarillo P, Elías-Zúñiga A, DíazElizondo JA, Gilkerson R, Lozano K. Past, present and future of surgical meshes: a review. Membranes (Basel). 2017; 7: 47.

11. Thanh Xuan N, Huu Son N. Laparoscopic transabdominal preperitoneal technique for inguinal hernia repair in adults. Cureus [Internet]. 2020 [Access August 02, 2020]; 12: e8692. Available in: https://doi.org/10.7759/cureus.8692

12. He C, Lu J, Ong MW, Lee DJK, Tan KY, Chia CLK. Seroma prevention strategies in laparoscopic ventral hernia repair: a systematic review. Hernia [Internet]. 2020 [Access August 03, 2020]; 24: 717-731. Available in: https://doi. org/10.1007/s10029-019-02098-1

13. Barbar S, Noventa F, Rossetto V, Ferrari A, Brandolin B, Perlati $M$ et al. A risk assessment model for the identification of hospitalized medical patients at risk for venous thromboembolism: the Padua Prediction Score. J Thromb Haemost [Internet]. 2010 [Access August 01, 2020]; 8:
2450-2457. Available in: http://pubmed.ncbi.nlm.nih. gov/20738765/

14. Barnes TG, McFaul C, Abdelrazeq AS. Laparoscopic transabdominal preperitoneal repair of spigelian herniaclosure of the fascial defect is not necessary. J Laparoendosc Adv Surg Tech A. 2014; 24: 66-71.

15. Baker JJ, Oberg S, Andresen K, Klausen TW, Rosenberg J. Systematic review and network meta-analysis of methods of mesh fixation during laparoscopic ventral hernia repair. Br J Surg. 2018; 105: 37-47. doi: 10.1002/bjs.10720. Epub 2017 Dec 11. PMID: 29227530.

Conflicto de intereses: Los autores declaran no tener ningún conflicto de intereses.

Declaración ética: Todos los procedimientos realizados en estudios con participantes humanos se hicieron de acuerdo con los estándares éticos de la institución y/o comités nacionales de investigación así como la declaración de Helsinki (revisada en 2013). 